Research article

\title{
Uncultivated fodder grass for cattle
}

\author{
R. Prameela ${ }^{1} *$ and M. Venkaiah ${ }^{2}$ \\ ${ }^{1}$ M.R.College for women, Vizianagaram, Andhra Pradeah, India \\ ${ }^{2}$ Department of Botany, Andhra University, Visakhapatnam, India \\ *Corresponding Author: prameelachris@yahoo.com \\ [Accepted: 25 September 2016]
}

\begin{abstract}
Vizianagaram district, one of the northern districts of Andhra Pradesh lies on the East Coast of India. The district is located between $17^{\circ}-15^{\prime}$ to $19^{\circ}-15^{\prime} \mathrm{N}$ and $83^{\circ}-00^{\prime}$ to $83^{\circ}-45^{\prime} \mathrm{E}$. Agriculture is the key occupation of the people of this district. Geographically vizianagaram district divided in to 3 regions, i.e 1) The hilly region 2) The plains and 3) The coasts. The present study has concentrated to generate the information on uncultivated fodder grass. The grass family Poaceae is of a major economic and ecological importance. It is the single most important family of flowering plants for survival of mankind. The grasses form a natural homogenous group of plants with remarkable diversity playing a significant role in the lives of human beings and animals. Studies on grasslands and wild grasses, especially of fodder value have become very important for development of dairy industry, productions of meat and restoration of degraded ecosystems. The grasses have good potentials in sustainable development of the country as well as conservation of both plant and animal diversity. Study areas in vizianagaram district are undisturbed places, open grounds, strip lands, unused rice fields, orchards and sandy areas. During rainy season grass seeds germinate and grow very fast. All these grasses are annuals. Where as in summer, we can see very little pasture. Many grasses are perennials; they can survive in drought conditions, because they possess thick rhizomatous or stoloniferous root system and tufted growth. Perennial grasses form a valuable pasture.
\end{abstract}

Keywords: Annuals - Herbivores - Pasture - Perennials - Strip lands - Vizianagaram.

[Cite as: Prameela R \& Venkaiah M (2016) Uncultivated fodder grass for cattle. Tropical Plant Research 3(3): 522-535]

\section{INTRODUCTION}

Hooker's 'Flora of British India' accounted for the known grasses of the country (Hooker 1896). Cooke (1901-1908) provided an account of grasses along with other families in his 'Flora of the Presidency of Bombay'. Gamble (1896) published 'The Bambuseae of British India'. Blatter \& McCann (1935) published an excellent illustrated account of Bombay grasses while Achariyar \& Mudaliyar (1921) published an account of South Indian grasses. Fischer (1934) contributed account for the grasses of Madras Presidency. Bor (1960) made extensive studies on grasses of Assam, Uttar Pradesh and published some 125 papers on Indian grasses and finally published a consolidated concise account of grasses of the whole Indian subcontinent 'The grasses of Burma, Ceylon, India and Pakistan. Several studies were conducted on the flora of North Coastal Andhra Pradesh. Subba rao (1977) studied on the flora of Visakhapatnam; Sriramulu (1986) studied flora of Srikakulam and Venkaiah (2004) flora of Vizianagaram district. In the present studies an attempt made to study the uncultivated fodder grasses of Vizianagaram district.

Everybody knows that all herbivorous animals depend upon the grass for their food. We can differentiate herbivorous animals into two categories namely, wild and domesticated or livestock animals. We need not take care about wild herbivores, because they feed on wild grass or green pastures of the forest areas. But we have to take a special care for livestock. Cultivated grass is compulsory for feeding the livestock at home. Generally cultivated grass is growing at the farms for livestock. It is very expensive and limited. In Vizianagaram there are no grasslands, but farmers are growing some types of grasses like Pennisetum spp., Sorghum spp., and also used straw of Rice, Maize, Sugarcane, Finger millet, Proso millet etc. It is limited and not sufficient for livestock, that's why they are being taken to open areas, where there are some pastures available. The present study concentrated on these uncultivated grasses growing in open lands and used as fodder. 


\section{MATERIALS AND METHODS}

Study areas in vizianagaram are undisturbed places, open grounds, Striplands, unused rice fields, orchards and Sandy areas. During rainy season grass seeds germinate and grow very fast. All these grasses are annuals. Examples of annual grasses are Brachiaria spp, Digitaria, Echinochloa, Eriochloa, Ischaemum, Paspalidium, Rottboellia, Setaria, Sorghum, Sporobolus and Urochloa etc. These grasses collectively form in a good pasture for the cattle. Where as in summer we can see very little pasture. Many grasses are perennials, they can survive in drought conditions, because they possess thick rhizomatous or stoloniferous root system and tufted growth. Perennial grasses form a valuable pasture. Examples of perennial grasses are Aristida, Chloris, Chrysopogon, Cenchrus, Cynodon, Dactyloctenium, Dichanthium, Eragrostis, Heteropogon, Paspalum, Paspalidium, Perotis and Themeda etc.

Regular field trips have been undertaken to the study areas of the district and collected the uncultivated grasses. The collected grasses are identified with the help of regional floras and Herbarium, BSI, Coimbatore.

\section{RESULTS}

\section{Enumeration of Species}

1. Alloteropsis cimicina (L.) Stapf in Prain, Fl. Trop. Afr. 9: 487.1919; GS. 357; Bor 276; chowdhary in Ind. For. 86: 90.1960; Gam vol. 3.1766; MV. 197; V. Fl. 213; HS. 52.1972.

Milium cimicinum L., Mant. Pl. 2: 184.1771.

Herbs, annuals, tufted, decumbent, hairy with bulbous based hairs; nodes hairy, internodes smooth leafy sheaths, leaf blades hairy, $7 \mathrm{~cm}$ long, 1-1.3 cm wide, ovate lanceolate, base cordate; base of the internodes purple colour; spikes $4-5$, terminal $6 \mathrm{~cm}$ long, 20-24 spikelets in each spike. Spikelets $0.3 \mathrm{~cm}$ long, elliptic, green, upper glume ciliate, acuminate, upper lemma aristate, keeled, upper palea membranous contains bisexual floret, lower glume mucronate, smaller than the upper one, lower lemma 3 nerved, shortly mucronate and its palea smaller membranous, contains malefloret, stamens 3.

2. Aristida adscensionis L., Sp. Pl. 82.1753; FBI 7: 221; var. adscensionis Bor 407.

A. depressa Retz., Obs. Bot 4. 22.1786; GS. 360; AP. Fl. 1130; Gam vol. 3: 1809.

Annual, tufted, branched, grows on rocky places; culms erect or decumbent, slender, rootstock creeping, nodes glabrous, leaf blades very narrow or linear; inflorescence contracted panicle, spikelets single, pedicel scaberulous, callus present, glumes 2 unequal, aristate, keel spinuscent, lemma base fringe of hairy, awns 3, unequal, stamens 3 , stigmas plumose.

3. Aristida funiculate Trin. \& Rupr., Sp. Gram. Stip. 159.1842; var. funiculate; Bor 410.1960; Gam vol. 3: 1809; FBI 7: 226.1896.

An annual, tufted grass, culms upto $50 \mathrm{~cm}$ tall, geniculately ascending; nodes glabrous, ligule a small ciliate membrane, blade 6-16 $\times 0.1-0.2 \mathrm{~cm}$ linear convolute, base cordate, apex acuminate; inflorescence panicle, lax, narrow, spikelets single, pedicelled, 1-flowered, glumes linear, hyaline, lemma truncate, awn trifid, subequal, stamens 3, caryopsis spiny.

4. Aristida hystrix L.f., Suppl. Pl. 113.1781; FBI 7: 225.1896; Gam vol. 3.1809; Bor 410; AP. Fl. 1131. (Fig. 1A)

Annual, small herbs upto $30 \mathrm{~cm}$ high; stems shows pseudo dichotomous branching, branches spreading; leaves small, linear, ligule gringe of hairs, axis angled, axis and nodes hairy; inflorescence panicle, effuse, dichotomously branches, spikelets single, pedicelled; glumes unequal, aristate, awns 3, double the length of the spikelets, stamens 3, base of the spikelet black coloured, dry spikelets straw coloured.

5. Bothriochloa pertusa (L.) A. Camus., Soc. Lyon. 1930, Gam vol. 3.1731; Bor 109.1960.

A perennial, stoloniferous grass, culms upto $50 \mathrm{~cm}$ tall, ascending, nodes sparsely hairy, leaf sheaths $2-6 \mathrm{~cm}$ long, glabrous, ligule membranous, blades 3.0-10 $\times 0.2-0.4 \mathrm{~cm}$, linear, base truncate, apex acuminate, glabrous; racemes 2-6, $5 \mathrm{~cm}$ long, slender, digitate, silky hairy, spikelets $6 \mathrm{~mm}, 2$-nate, pitted, sessile spikelet: $4 \mathrm{~mm}$, oblong-lanceolate, pitted, callus bearded, lower glume elliptic-oblong, bearded below, dorsally pitted, subchartaceous, upper glume ovate-lanceolate, finely pointed at the tip, lower lemma lanceolate, nerveless, hyaline, upper lemma reduced to a slender awn, stamens 3, stigmas plumose, caryopsis oblong, pedicelled spikelet: $4 \mathrm{~mm}$, male or barren, similar to sessile, spikelet, but awnless.

www.tropicalplantresearch.com 
6. Brachiaria reptans (L.) C.A. Gardner \& C.E. Hubb., Hooker's Icon. Pl. 34: t. 3363.1938; Fl. Trop. Afr. 9: 601.1920 .

Annuals, culms prostrate or creeping, slender, branched; leaves ovate-lanceolate, cordate at base, margin scaberulous, mouth ciliate, ligule a fringe of hairs, leaf sheath clasping the stem, shorter than the internode; inflorescence panicle, branches up to 9; spikelets 2-nate, both are pedicelled, bears 4-5 setae.

7. Capillipedium assimile (Steud.) A.Camus., Fl. India 15: 6.1984. (Fig. 1B)

Andropogon assimillis Steud. in Zoll. Syst. Verz. 48.1854. et in Syn. Pl. Glum. 1: 397.1854; FBI 7: 179.1896.

Capillipedium assimile (Steud.) Camus in Lecomte, Fl. Gen. del' Indo-chine 7: 314.1922; Bor 110.1960.

C.glaucopsis (Steud.) Stapf in Hook., lc. Pl. Sub. tab. 3085.1922; Gam vol. 3. 1730.

A perennial, tufted grass, culms upto $50 \mathrm{~cm}$ long, decumbent, often branched, nodes glabrous or bearded, leaf sheaths usually glabrous, ciliate at mouth, ligule a short membrane, apex ciliate, blades 8-20 × 3-6 cm, linear-lanceolate, apex acuminate, slightly hairy; panicle lax, branches slender, capillary, with long hairs on the axils, spikelets few, distant, joints sparsely ciliate, sessile spikelet: $3 \mathrm{~mm}$, lower glume oblong, ciliate, not pitted, upper glume lanceolate, lower lemma linear, shorter, obtuse, upper lemma reduced to a scale, flattened based awn, pedicelled spikelet: $4 \mathrm{~mm}$, not awned, pedicels sparsely ciliate, lower glume lanceolate, lower lemma obovate-oblong, apex ciliate, lower glume lanceolate, lower lemma obovate-oblong, apex ciliate, hyaline, upper lemma absent, stamens 3 .

8. Cenchrus ciliaris L., Mant. Alt. 302.1771; Bor 287.

Pennisetum cenchroides Rich. in Pers., Syn. Pl. 1: 72.1805, nom. Superfl. FBI 788.1896; AP Fl. 1146; Gam vol. 3.1793; Maha Fl. 421.

Tufted herbs, erect or decumbent; leaves linear with tubercle based hairs; racemes cylindric, dense, pale purple, bristles connate at base only, scabrid; involucral spikelets 2, unequal, 2-flowered, large spikelet contains bisexual and male florets, small spikelet contains 2 male florets; stamens 3, green.

9. Chloris barbata Sw., Fl. Ind. Occ. 1: 200.1797; Bor 465; FBI 7: 292; Gam vol. 3.1836; AP Fl. 1148; HS. 508; MV. 199. (Fig. 1C)

Annual tufted herbs, base creeping; leaves linear-lanceolate, tip acuminate, ligule membranous; racemes umbellate, spikes 8-12, rachis scaberulous, spikelets 3-flowered, perfect floret 1, glumes linear, densely ciliate on the margin; fertile lemma obovate, densely stiff ciliate on margins, awned; both sterile lemmas boat-shaped, awned, stamens 3, caryopsis oblong, compressed.

10. Chrysopogon aciculatus (Retz.) Trin., Fund. Agrose. 188.1820; Bot. Bihar \& Orissa 1035.1924; Gam vol. 3. 1738.1934; Bor 115.1960; V Fl. 209. (Fig. 1D)

Andropogon aciculatus Retz., Obs. Bot. 5: 22.1789; FBI 7: 188.1896; AP. Fl. 1155.

Perennials with creeping rhizomes, culms decumbent, glabrous, leaf blades sub basal, upto $7 \mathrm{~cm}$ long, 0.5 cm wide, glabrous; inflorescence panicle, branches in whorled, in each whorl 5-6 spikes, each spike has long peduncle, tip of the peduncle has 3 spikelets, middle one sessile, remaining two pedicelled, sessile spikelet base hairy awned, bisexual, lower glume bidentate, 3-nerved, 2-keeled, upper glume awned, ciliate, lower lemma epaleate, empty, upper lemma aristate, paleate, contains bisexual floret.

11. Chrysopogon orientalis (Desv.) A. Camus., Bor 118.1960. (Fig. 1E)

Rhaphis orientalis Desv., Opuse. 69.1831.

Andropogon wightianus Nees ex Steud., Syn. Pl. Glum. 1: 395.1854. FBI 7: 191.1896; AP. Fl. 1155; Gam vol. 3. 1736.

A perennial, tufted grass, culms upto $80 \mathrm{~cm}$ tall, slender ascending at base, nodes glabrous, leaf sheaths 5-9 $\mathrm{cm}$, shortly ciliate-glabrous at mouth, ligule short, villous; inflorescence panicle, $15 \mathrm{~cm}$ long, whorled branches, branches with reddish-brown hair tip, spikelets 3, middle sessile lateral 2 pedicelled, 3 spikelets awned; peduncle very long, slender, spiral, tip hairy, sessile spikelet, lemma awn, very long, as long as peduncle, glume awn as long as spikelet, sessile spikelet contains bisexual floret, pedicelled spikelet lanceolate, pubescent pedicel truncate, margins shortly ciliate, glumes bright red/purple, lower glume ciliate awned, upper glume lanceolate, acuminate, lower lemma, upper lemma also ciliate, staments 3.

12. Cynodon dactylon (L.) Pers., Syn. Pl. 1: 85.1805; FBI 7: 288.1960; Gam vol. 3.1835; Bor 469. www.tropicalplantresearch.com 
Panicum dactylon L., Sp. Pl. 58.1753.

Perennial, stoloniferous, culms creeping or decumbent; leaves small, linear, base rounded, tip acuminate, distichous; spikes 4-5, digitate; spikelets sub sessile, laterally compressed; glumes sub equal, lanceolate, 1nerved, lemma oblong, aristate, keels ciliate, palea linear, stamens 3, caryopsis oblong.

13. Dactyloctenium aegyptium (L.) Willd., Ess. Agrost. Expl. t. 15.1812; Gam vol. 3: 1840 (1273). 1934; Bor 489.1960. (Fig. 1F)

Cynosurus aegypticum L., Sp. P1. 72.1753.

Eleusine aegyptia (L) Roxb., Fl. Ind. 1: 345.1820; FBI 7: 295.1896.

Dactyloctenium aegyptiacum Willd., Enum. Pl. Hort. Berol. 1029.1809.

An annual grass, culms erect or creeping, roots at the nodes, branches geniculately ascending, nodes bearded or glabrous, leaf sheaths 2-6 cm long, hairy near mouth, ligule a ciliate rim, blades $2-10 \times 0.3-0.8 \mathrm{~cm}$, linear, flat, base rounded, apex acuminate, glabrous or hairy with bulbous hairs; spikes 2-6, digitately radiating, upto 7 cm long, spikelets 3-5 flowered, laterally compressed, sessile, glumes subequal, folded, in lower keels scabrid, in upper keel smooth or hispid, awned, lemmas aristate, palea hyaline, winged on keels, stamens 3, stigmas plumose; caryopsis obovate.

14. Dichanthium annulatum (Forssk.) Stapf., Fl. Trop. Afr. 9: 178.1917; Gam vol. 3.1740; Bor 133.1960; Deshp in Fasc. Fl. India 15: 5.1984.

Andropogon annulatus Forssk., Fl. Aegy-Arab. 173.1775; FBI 7: 196.1896.

A perennial, tufted grass, culms upto $70 \mathrm{~cm}$ tal, erect or ascending, nodes bearded, leaf sheaths $5-10 \mathrm{~cm}$ long, glabrous, ciliate near the mouth, ligule membranous, blades 6-20 $\times 0.3-0.5 \mathrm{~cm}$, linear, base rounded, apex acuminate; racemes $6 \mathrm{~cm}$ long, sub digiatately fascicled, peduncles glabrous, spikelets $4 \mathrm{~mm}$, 2-nate, sessile spikelet: 4mm, bisexual, elliptic-oblong, lower glume elliptic-oblong, truncate, keeled, ciliate, upper glume, nerveless, upper lemma narrow, with a scabrid, slender awn, stamens 3, stigmas plumose, caryopsis oblongslightly compressed, pedicelled spikelet: $4 \mathrm{~mm}$, male or barren, lower glume elliptic-oblong, 7-11 nerved keeled, upper glume narrow, 3-nerved, lower lemma ciliate, upper lemma small or obsolete.

15. Dichanthium caricosum (L.) A. Camus., Gam vol. 3. 1741; Bor 134.1960; Deshp. in Fasc. Fl. India 15: 7.1986.

Andropogon caricosus L., Sp. Pl. 2: 1480. 1763; FBI 7: 196.1896.

A perennial, tufted grass, ascending, nodes bearded or with short hairs, leaf sheaths $3-10 \mathrm{~cm}$ long, glabrous, ligule shortly ciliate membrane, blades $4-20 \times 0.3-0.5 \mathrm{~cm}$ linear, base rounded, apex acuminate, glabroussparsely pubscent, racemes $1-6$, upto $8 \mathrm{~cm}$ long, both terminal and axillary; joints and pedicels of racemes with short hairs, spikelets 4mm, 2-nate, sessile spikelets: 2mm, oblong, bisexual, lower glume oblong-obvate, 4nerved, truncate, pilose, upper glume elliptic, 3-nerved, hairy, lower lemma lanceolate, nerveless, hyaline, upper narrow, awned, stamens 3, pedicelled spikelet, lower glume obovate, many nerved, pilose, upper glume and lemmas similar to sessile spikelet.

16. Digitaria ciliaris (Retz.) Koeler., Descr. Gramin. 27.1802. (Fig. 1G)

Digitaria marginata Link var. fimbriata (Link) Stapf in Prain, Fl. Trop. Afr. 9: 440.1919; Gam vol. 3 : 1764 (1222). 1934

D. adscendens (H.B.K.) Henr. in Blumea 1: 92.1934; Bor 298.1960.

Panicum adscendens H.B.K. Nov., Gen. Pl. 1: 102.1821.

An annual, tufted grass, culms upto $90 \mathrm{~cm}$ tall, usually ascending from geniculate or prostrate base, branching from lower nodes, nodes glabrous, leaf sheaths $4-12 \mathrm{~cm}$ long, glabrous, ligule membranous, truncate, blades 4-10 × 0.4-0.6 cm, linear-lanceolate, base cordate, apex acute, glabrous, racemes 4-9, $10 \mathrm{~cm}$ long, digitate, rachis serrate, trigonous, spikelets $3 \mathrm{~mm}$, elliptic-lanceolate, 2-nate, apperssed to the rachis, glabroussparsely ciliate, homomorphous, lower glume reduced to a triangular scale, upper glume linear-lanceolate, 3-5 nerved, pubescent, lower lemma oblong-lanceolate, 5-nerved, marginal nerves pubescent, upper lemma oblonglanceolate, obscurely 3-nerved, stamens 3, stigmas plumose, caryopsis oblong.

17. Digitaria longiflora (Retz.) Pers., Gam vol. 3: 1765; Bor 302.1960.

Paspalum longiflorum Retz., Obs. Bot. 4: 5.1786; FBI 7: 17.1896. 

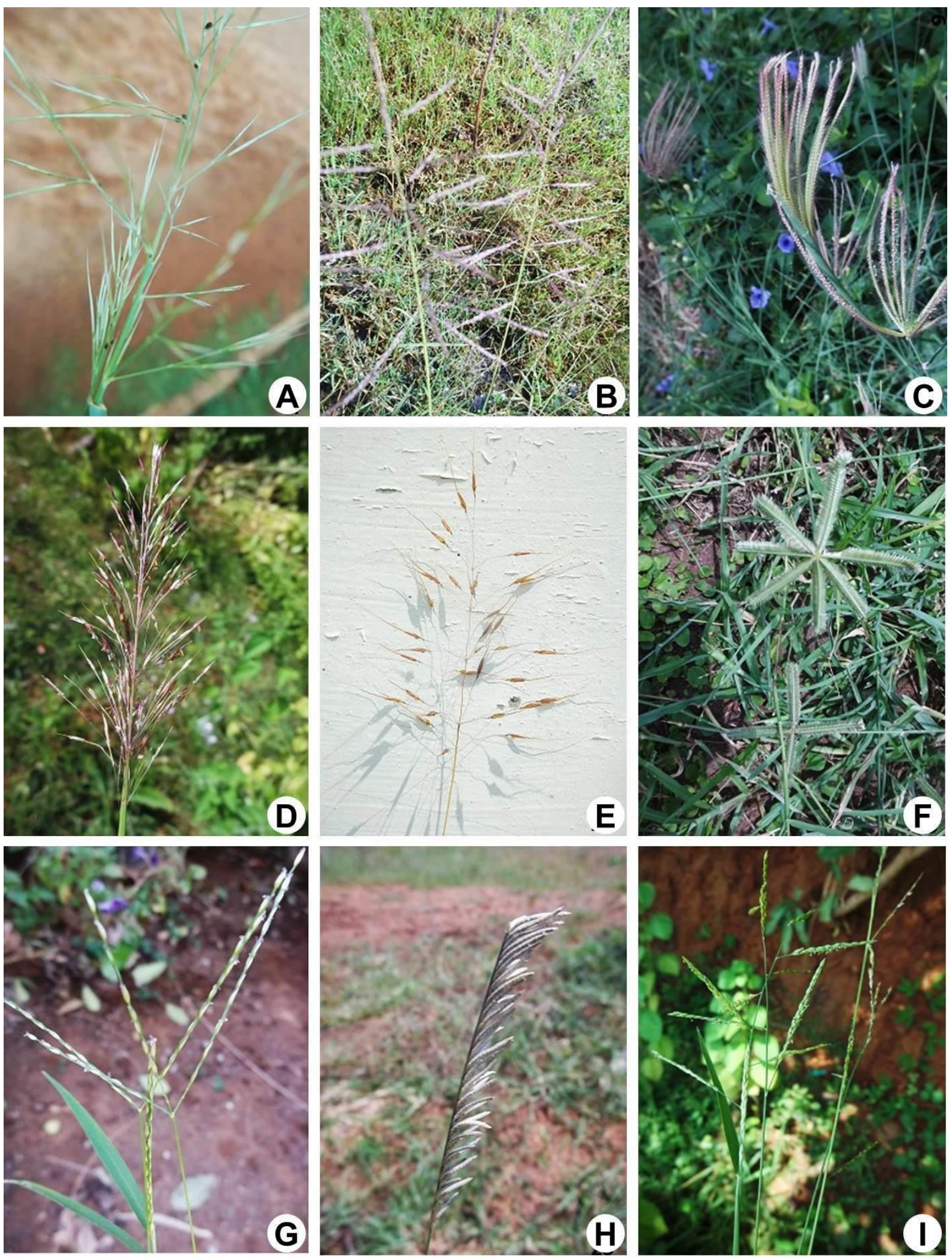

Figure 1. A, Aristida hystrix L.f.; B, Capillipedium assimile (Steud.) A. Camus.; C, Chloris barbata Sw.; D, Chrysopogon aciculatus (Retz.) Trin.; E, Chrysopogon orientalis (Desv.) A. Camus.; F, Dactyloctenium aegyptium (L.) Willd.; G, Digitaria ciliaris (Retz.) Koeler.; H, Eragrostiella bifaria (Vahl) Bor.; I, Eriochloa procera (Retz) C.E. Hubb.

An annual, tufted grass, culms upto $60 \mathrm{~cm}$ tall, ascending, rooting at the nodes, nodes glabrous, leaf sheaths 2-4 cm long, glabrous, ligule membranous, blades $2-7 \times 0.2-0.5 \mathrm{~cm}$, linear-lanceolate, base rounded, apex acuminate, glabrous, racemes $2-5,7 \mathrm{~cm}$ long, digitate, rachis serrate, spikelets $2 \mathrm{~mm}$ long, elliptic, homomorphous, sparsely pubscent, lower glume absent, upper glume oblong-ovate, 3-5 nerved, hairy, lower www.tropicalplantresearch.com 
lemma similar to upper glume, upper lemma ovate-oblong, subchartaceous, nerveless, palea similar to upper lemma, stamens 3, stigmas plumose, caryopsis ellipsoid.

18. Digitaria sanguinalis (L.) Scop., Fl. Carn. ed. 2.1. 552.1772; Bor 304.1960; T.A. Cope in Nasir \& Ali Fl. Pak. 143. 231.1982.

Panicum sanguinalis L., Sp. Pl. 57.1753.

Paspalum sanguinale (L.) Lam., III. 1: 176.1791; FBI 7: 13.1896.

Digitaria sanguinalis (L.) Scop. ssp. aegyptica var. frumentacea Henr. Monogr.

Digitaria 985.1950; Bor 304.1960.

Digitarua sabguinalis ssp. vulgaris var. rottleriana Henr., 1.c. 986; Bor 304.1960.

An annual, tufted grass, culms up to $40 \mathrm{~cm}$ tall, erect or ascending from a creeping, branching base, leaf sheaths glabrous, hairy near the mouth, blades $2.5-18 \times 0.4-0.8 \mathrm{~cm}$, linear; spikes, slender, 3-10, $8 \mathrm{~cm}$ long, digitate, spikelets 2-nate on abbreviated peduncles, imbricate, lower glume 3-5 nerved, lateral nerves marginal, lower lemma lanceolate or oblong-lanceolate, smooth; caryopsis oblong, whitish.

19. Dinebra retroflexa (Vahl) Panz., Gam vol. 3: 1841 (1274). 1934; Bor 491.1960.

Cynosurus retroflexus Vahl, Symb. Bot. 2. 20.1791.

Dinebra arabica Jacq., Fragm. Bot. 77. t. 121. f. 1.1807; FBI 7: 297.1896; AP. Fl. 1182.

An annual, tufted grass, culms up to $70 \mathrm{~cm}$ tall, geniculately ascending below, nodes glabrous, leaf sheaths 5-9 cm long, loose, glabrous, ligule narrow membrane; blades 5-10 × 0.2-0.4 cm linear, base cordate, apex acuminate, glabrous, spikes racemosely arranged along the axis of an inflorescence, up to $20 \mathrm{~cm}$ long, rachis stiff, serrate, winged, spikelets 5mm, alternate, 2-flowered, glumes persistent, lanceolate, 1-nerved, strongly keeled, slightly recurved, scaberulous awn, lemma ovate-oblong, hyaline; palea ovate, hyaline, 2-keeled, minutely sparsely ciliate on the keel, stamens 3, stigmas plumose, caryopsis ellipsoid.

20. Echinochloa colonum (L.) Link., Gam vol. 3: 1776; Bor 308.1960.

Panicum colonum L., Syst. Nat. (ed.10) 810.1759; FBI 7: 32.1896.

An annual, tufted grass, culms up to $70 \mathrm{~cm}$ tall, branching from lower nodes, nodes glabrous, leaf sheaths 5$15 \mathrm{~cm}$ long, glabrous, ligule obsolete, blade 5-25 ×0.3-0.6 cm, linear-lanceolate, base cordate, apex acuminate, glabrous; spike like panicle, racemes 8-20, appressed to the rachis, rachis angular, scaberulous, spikelets 2.5 $\mathrm{cm}$, broadly ovate, acute or sub-cuspidate, crowded in rows, second, glumes unequal, lower glume half the length of the upper glume, broadly ovate-orbicular, upper glume broadly ovate, mucronate, puberulous, lower lemma broadly ovate, cuspidate, upper lemma broadly ovate, obtuse, palea oblong, stamens 3, stigmas plumose; caryopsis broadly ellipsoid.

21. Echinochloa crusgalli (L.) Gam, vol. 3: 1777 (1231). 1934; Bor 310.1960.

Panicum crusgalli L., Sp. Pl. 56.1753; FBI 7: 30.1896.

An annual, tufted grass, culms $70 \mathrm{~cm}$ tall, branching from lower nodes, nodes glabrous, leaf sheaths 5-25 $\mathrm{cm}$, glabrous, ligule obsolete, blades 5-20 ×0.4-0.8 cm, linear-lanceolate, base cordate, apex, acuminate, glabrous; spikelike panicle $10 \mathrm{~cm}$ long, racemes spreading, rachis rtiquetrous, scabrid, spikelets broadly ovoid, 5 $\mathrm{mm}$, crowded, acute-cuspidate, awned, lower glume broadly ovoid or orbicular, margin ciliate, half as long as the upper glume, upper glume broadly ovate-oblong, concave, cuspidate, hispid on back, lower lemma ovateoblong, stamens 3 , stigmas plumose, caryopsis broadly ellipsoid.

22. Echinochloa frumentacea Link., Bor 311.1960.

Panicum crusgalli var. frumentaceum Hook.f. FBI 7: 31.1896.

Echinochloa colona Link var. frumentacea Ridl., Fl. Malay Penin 5: 223.1925; Gam vol. 3: 1777.

An annual, robust grass, culms up to $1 \mathrm{~m}$, leaf sheaths $12 \mathrm{~cm}$, ligule absent, blades to $20 \times 2 \mathrm{~cm}$, racemes 18 $\mathrm{cm}$, several seriate, spikelets $4 \mathrm{~mm}$, ovoid-broadly ellipsoid, hispid, glumes subequal, upper with a short mucronate apex, lower lemma mucronate, stiff-hispid outside, upper lemma crustaceous, short mucronate, palea similar to its lemma, caryopsis broadly ellipsoid.

23. Echinochloa stagnina (Retz.) Beauv., Gam vol. 3: 1777 (1231). 1934; Bor 311.1960.

Panicum stagninum Retz., Obs. Bot. 5: 17.1789.

P.crusgalli sensu Hook.f., FBI 7: 30.1896 p.p. non L. 1753.

www.tropicalplantresearch.com 
An annual stoloniferous grass, culms up to $50 \mathrm{~cm}$ tall, rooting at the nodes, nodes glabrous, leaf sheaths 5$10 \mathrm{~cm}$ long, loose, glabrous, ligule a fringe of stiff hairs, blades 4-15 $\times 0.4-0.8 \mathrm{~cm}$, linear-lanceolate, base cordate, apex acuminate, midrib prominent; spikelike panicle 5-10 cm long, racemes interrupted, rachis angular, scabrid, spikelets ovate, crowded, acutely acuminate, awned, secung, pubescent, lower glume broadly ovate, bidentate, pubescent, upper glume broadly ovate, acuminate, hairy at apex and margins, lower lemma ovate, hairy and scabrid at apex, awned, palea oblong-ovate, scabrid margin, male; upper lemma ovate, scabrid along the margins, acute, chartaceous, palea similar to upper lemma, bisexual, stamens 3, stigmas plumose; caryopsis broadly ellipsoid.

24. Enteropogon dolichostachya (Lag.) Keng., Gen. Sp. Pl. 5.1816; Gam vol. 3: 1838 ; Bor. 466.

Chloris incompleta Roth, Nov. Pl. Sp. 60.1821; FBI 7: 290.1896; Bot. Bihar \& Orissa 968.1924; Gam vol. 3.1838; Suppl. Bot. Bihar \& Orissa 164.1950; GS. 374; AP. Fl. 1149; MV. 199; Maha Fl. 426.

Slender perennials, upto $93 \mathrm{~cm}$ long, culms erect, sometimes decumbent, nodes glabrous, leaf blades linear acuminate, ligule comprise of long hairs, spikes 2-4 digitate, rhachis angled, scabrid on angles, spikelets unilateral, 2-flowered, 2-awned, 2 seriats, lower glume membranous 1-nerved, upper glume short awned, lower lemma membranous, 2-toothed at apex, awned, callus beareded.

\section{Eragrostiella bifaria (Vahl) Bor., FBI 7: 325.1896; Gam vol. 3.1828. (Fig. 1H)}

A perennial, tufted grass, culms up to $50 \mathrm{~cm}$ tall, slender, leaf sheaths glabrous, ligule a pubescent line, blades 5-20 × 1.0-1.5 cm, linear, filiform, base narrow, apex acute, glabrous, spike 5-25 cm long, spikelets $5 \mathrm{~mm}$ long, oblong, second, alternately arranged on the rachis, laterally compressed, 5-40 flowered, glumes subequal, deciduous, lanceolate, 1-nerved, keeled, lemma broadly ovate, 1-nerved, keeled, palea lanceolate, winged on keels, stamens 3, styles plumose; caryopsis globose.

26. Eragrostis cilianensis (All.) Janch., Gam vol. 3.1827; Bor 503.1960.

Poa cilianensis All., Fl. Pedem. 2: 246. t. 91. f. 2, 1785.

Eragrostis major Host., Icon. Descr. Gram Austr. 4: 14. t. 24.1809; FBI 7: 320.1896; HS. 516.

An annual, tufted grass, culms up to $90 \mathrm{~cm}$ high, leafy, branched, erect or geniculately ascending, nodes glabrous, leaf sheaths 2-4 cm long, loose ciliate at mouth, ligule a ciliate ridge, blades 5-12 $\times 0.4-0.6 \mathrm{~cm}$, lanceolate, flat, prominently nerved, base cordate, glandular along the margins, apex acuminate; panicle up to 20 cm long, spreading or contracted, rhachis stiff, spikelets $1 \mathrm{~cm}$ long, oblong, breaking up from down wards, 1030 flowered, glumes subequal, linear, ovate-lanceolate, scabrid on the nerves at back sides, lemma broadly ovate, acute, 3-nerved, palea linear, ciliate on the margins near the apex, persistent, stamens 3, stigmas plumose, caryopsis subglobose.

27. Eragrostis ciliaris (L.) R.Br., FBI 7:314.1896; Gam vol. 3.1825; Bor 506.1960.

Poa cilliaris L., Syst. Nat. ed. 10. 2. 875.1759; HS. 516.

An annual, tufted grass, culms up to $40 \mathrm{~cm}$ tall, slender, geniculately ascending below, nodes glabrous, leaf sheaths $2.5 \mathrm{~cm}$ long, glabrous, ciliate at the mouth, ligule ciliate, blades $2-5 \times 0.2-0.4 \mathrm{~cm}$, linear, convolute, base rounded, apex acuminate, glabrous, panicle up to $8 \mathrm{~cm}$ long, interrupted, cylindrical, branches short, spikelets $2.5 \mathrm{~mm}$, oblong, subsessile, glumes subequal, ovate-lanceolate, scabrid on the nerve on the backside, lemma ovate, 3-nerved, lateral nerves close to the margin, ciliate, palea obovate, truncate, with soft ciliate, stamens 3, stigmas plumose, caryopsis ovoid.

28. Eragrostis coarctata Stapf., FBI 7: 313.1896; Gam vol. 3.1825; Bor 507.1960; GS. 396.

Annual, tufted, 3-4 culms in a tuft, erect, slender, nodes, internodes glabrous, leaf sheath, leaves hairy; panicle dense 15-20 cm long, grayish-purple, spikes up to 15, short 5-6 cm long, villous at nodes, spikelets up to 50 arranged in distichous manner, pedicel short scaberlous, spikelets single contains a bisexual floret, glumes unequal, scaberulous on margins, tip acute or acuminate, 1-nerved, lemma \& palea ovate, tip obtuse, margins ciliate, pink, stamens 3, lodicules 2, grain oblong, reddish brown.

29. Eragrostis japonica (Thunb.) Trin., Gam vol. 3.1826; Bor 509.1960.

Poa japonica Thunb., Fl. Jap. 51.1784.

Eragrostis interrupta Beauv. var. tenuissima Stapf in. FBI 7: 316.1896; GS. 397. 
Perennial, tufted grass, culms 1.0-1.5 m high, geneculately ascending at base, plants glabrous, ligule membranous, leaf blade linear-lanceolate, tip acuminate, base cordate or rounded; panicle up to $50 \mathrm{~cm}$ long, branches whorld, lax, inturrepted, internodes long, spikelets 2-9 flowered, glumes subequal, narrow, 1-nerved, lemma 3-nerved, palea boat shaped, scaberulous, stamens 3 or 2, caryopsis obovoid.

30. Eragrostis tenella (L.) Beauv. ex Roem. \& Schult., FBI 7: 315.1896; Bor 513.1960. var. tenella; HS. 518; GS. 398.

Annual, densely tufted grass, culms $50 \mathrm{~cm}$ high, leaf sheaths glabrous long ciliate at mouth, panicle $20 \mathrm{~cm}$ long, decompound, spreading or contracted, spikelets 3-9 flowered, small, palea keeled ciliate, caryopsis ovate.

31. Eragrostis viscosa (Retz.) Trin. Acad. Sci. Petersb. 6, 1: 397.1830; Gam vol. 3.1826; Bor 515.1960. Poa viscose Retz., Obs. Bot. 4: 20.1786/87.

Eragrostis tenella var. viscosa (Retz.) Stapf in FBI 7: 315.1896.

Perennial, tufted, viscid, scented grass, culms $50 \mathrm{~cm}$ high, glandular patches scattered throughout, nodes glabrous ligule fimbriate membrane, panicle $20 \mathrm{~cm}$ long, cylindric, dense glandular, branches numerous, spreading spikelets 5-20 flowered, glumes unequal, linear, 1-nerved, lemmas ellipsoid, palea linear, ciliate on the keels, caryopsis ovoid.

32. Eriochloa procera (Retz) C.E. Hubb., Gam vol. 3.1767; Bor 312; AP. Fl. 1202. (Fig. 1I) Eriochloa polystachya Hook.f., FBI 7: 20.1896; Maha Fl. 494.

Perennials, densely tufted, up to $1 \mathrm{~m}$ high, root stock short; leaves linear, base cordate, ligule rim of hairs.

33. Eriochloa fatmensis (Hochst. \& Steud.) Clayton., Bor 312; Maha Fl. 493.

Annual, terrestrial, culms 30-60 cm high, geneculately ascending and rooting at lower nodes, ligule hairy; panicle of simple or branched racemes, 3-10 cm long; spikelets paired or rarely solitary, ellipsoid, hispid.

34. Heteropogon contortus (L.) Beauv. ex Roem. \& Schult., Gam vol. 3: 1743; Bor 163.1960. (Fig. 2A) Andropogon contortus L., Sp. Pl. 1045.1753; FBI 7: 199.1896; AP. Fl. 1206.

An annual, tufted grass, culms up to $60 \mathrm{~cm}$ high, erect or decumbent below; nodes glabrous, leaf sheaths 5$10 \mathrm{~cm}$, glabrous; ligule membranous, fimbriate; blades $4-10 \times 0.2-0.4 \mathrm{~cm}$, lanceolate, flat, base rounded, apex acute, scaberulous; racemes solitary, $6 \mathrm{~cm}$, awns forming a twisted spire; lower spikelets homogamous, unawned, either neuter or male; upper spikelets heterogamous, awned, 2-nate, sessile hermaphrodite, pedicelled male or barren; pedicel glabrous; callus pungent, bearded with brown hairs; sessile spikelets: $7 \mathrm{~mm}$, linear, hispidulous, female; callus bearded with brown hairs, lower glume linear-oblong, hispidulous; upper glume lenear, obtuse, margin hyaline, dark brown, hispidulous; lower lemma oblong, truncate, epaleate; upper lemma linear, stipitoform, awned, epaleate; stamens 3, stigma plumose, caryopsis linear; pedicelled spikelets: oblong, hispidulous, lower glume lanceolate, dorsally hispidulous with long bulbous based hairs, winged, serrulate; upper glume oblong-lanceolate, margin hyaline; lemmas hyaline, nerveless, hairy.

35. Oplismenus burmanni (Retz.) P. Beauv., FBI 7.66; Gam vol. 3: 1777; V. Fl. 216.

Panicum burmanii Retz., Obs. 3. 10.1783; MV.205; HS. 521; GS. 412.

A small procumbent herb, lower nodes bears roots, leaf sheaths, leaf blades hairy, leaf blade ovate lanceolate, base cordate, ligule fringe of hairs; inflorescence panicle, racemes 4, distant, rhachis trigonous, villous, spikelet 2 nate, unequal, sessile spikelet small, pedicelled spikelet large, pedicel villous, 2 flowered, lower glume membranous, ovate, obtuse, pubescent margins ciliate, 3 nerved, awned, awn scaberulous, upper glume similar to lower glume 7 nerved, awn short, lower lemma awned, empty, membranous, 9 nerved, 2 keeled, epaleate; upper lemma chartaceous, its palea similar, contains bisexual floret, stamens 3 , stigmas plumose.

36. Oplismenus compositus (L.) P. Beauv., FBI 7: 66; Bor. 317; S. 317; Gam vol. 3.1777; Bot. Bihar \& Orissa 3.1045; MV. 205; HS. 521.

Pannicum compositus L., Sp. Pl. 1: 57.1753.

Oplismenus lanceolatus Kunth, Rev. Gram. 45.1829. 
Perennial, stoloniferous up to $60 \mathrm{~cm}$ tall, creeping, rooting at nodes, nodes glabrous, leaf sheath $2-9 \mathrm{~cm}$ long, margin density liliate, ligule fimbriate membrane; inflorescence terminal, racemes $20 \mathrm{~cm}$ long, spikelets 2 flowered, awned, lower glume awned, upper glume aristate.

\section{Panicum maximum Jacq., Gam vol. 3.1783; Bor 327.}

Perennial tufted grass, culms up to $1 \mathrm{~m}$ high, stout, nodes hirsute; ligule membranous, blades linear, flat, base cordate, margin scaberulous, apex acuminate, glabrous, mid rib prominent; panicle lax, spikelets linearoblong, dense, lower glume orbicular, half the length of spikelet, 1-nerved, upper glume broadly oblong, margins incurved, 5-nerved; lower lemma oblong, 5-nerved, palea oblong, 2-nerved, male, upper lemma ovate oblong, 3-nerved, transversely rugose, it's palea similar, nerve less, bisexual.

\section{Panicum notatum(Retz.) Bor, 329; Gam vol. 3.1783.}

Perennial slender grass, culms up to $1 \mathrm{~m}$ high, erect from woody stock, branched; nodes glabrous; leaf sheaths ciliate on margin, ligule of soft long hairs, blades linear-oblong, base amplexicaul, margin with tubercled based hairs, apex acuminate, pubescent on both surfaces; panicle up to $25 \mathrm{~cm}$ long, lax, open, branches spreading, spikelets few, pedicels very long, filiform; lower glume ovate, 3-5 nerved, pilose, upper glume broadly ovate, 5-nerved; lower lemma ovate, 5-nerved, hairy on outsite, epaleate, upper lemma ovate, crustaceous, indurated, palea coriaceous with involute margins.

39. Paspalidium flavidum (Retz.) A. Camus., Fl. Indo-Chine 7.419.1922; Bot. Bihar \& Orissa 1000.1924; Gam vol. 3.1774; Bor 333.1960. (Fig. 2B)

Panicum flavidum Retz., obs. Bot. 4: 15.1786; FBI 7. 28.1896; GS. 419; MV. 206; HS. 523; V. Fl. 214.

Perennials, tufted, up to $80 \mathrm{~cm}$ high, culms erect base decumbent, striate, glabrous, nodes glabrous, leaf blades linear, lanceolate, acute or obtuse, cordate at base, margins scabrid, ligule membranous, base narrow ciliate, sheaths glabrous, striate; inflorescence racemose, racemes distant, up to $2 \mathrm{~cm}$ long, rhachis flattened, minutely ciliate, spikelets biseriate, sub sessile, spikelets 2 flowered, lower floret empty, upper floret bisexual, lower glume hyaline, suborbicular, 3-nerved, lower lemma ovate-oblong, subcoriaceous, 5- nerved, palea sililar to its lemma, bidentate, upper glume membranous, sub orbicular, rounded at apex, 7-nerved, upper lemma ovate, acute, coriaceous, granulose, palea similar to its lemma 2 keeled, lodicules 2, broadly cuneate, stamens 3 , yellow, ovary triquetrous, styles 2, laterally exserted.

40. Paspalidium geminatum (Forssk.) Stapf., Fl. Trop. Africa 9.583.1920; Bot. Bihar \& Orissa 1001.1924; Gam vol. 3.1774.1934; Susppl. Bot. Bihar \& Orissa 176.1950; Bor. 333.1960.

Panicum geminatum Forssk., Fl. Heg-Arab. 18.1775.

P. paspaloides Pers., Syn. 1. 81.1805; FBI 7: 30.1896; GS. 420.

Perennial, culms up to $50 \mathrm{~cm}$ high, creeping below, branches from the lowernodes, nodes glabrous, ligule a ciliate rim; panicle, spike like, racemes 10-15, alternate on the rhachis; spikelets solitary, 2- flowered, lower male, upper bisexual.

41. Paspalidium punctatum (Burm.) A. Camus., Gam vol. 3.1774; Bor 333. Panicum punctatum Burm., f. Fl. Ind. 26.1768; FBI 7: 29.1896; AP. Fl. 1233.

A perennial, tufted grass, culms up to $1 \mathrm{~m}$ tall, prostrate, rooting at lower nodes, spongy, leaf sheaths $10 \mathrm{~cm}$ long, ligule a ridge of hairs, blades $10-15 \times 0.5-1.0 \mathrm{~cm}$, flat or convolute, apex acuminate, glabrous; spike like panicle $30 \mathrm{~cm}$ long, racemes $20 \mathrm{~cm}$ long, distant, sessile, compressed, appressed to the rachis, spikelets $3 \mathrm{~mm}$ long, 18-40 per raceme, ovoid, lower glume truncate; upper glume broadly oblong; lower lemma acuminate, palea empty, upper lemma punctate, apiculate, palea coriaceous, margins incurved, stamens 3 , stigmas plumose, caryopsis ovoid.

42. Paspalum canarae (Steud.) Veldkamp., Blumea 21: 721973.

Panicum canarae Steud., Syn. Pl. Glumac. 1: 58.1853.

Paspalum compactum sensu FBI 7: 12.1896, non Roth 1821; Gam. vol. 3.1772; Bor 336.1960; GS. 421.

Annuals, slender, tufted, culms decumbent glabrous nodes bearded, leaf blades ovate-lanceolate, obtuse, base rounded, hirsute with bulbous based hairs, margins ciliate ligule a rim of hirsute hairs, inflorescence racemose, rhachis angled, winged, hairy at the base of branches, spikelets 2-nate, plano convex, lower glume 
absent, lower lemma empty as long as the spikelet, 3-nerved epaleate, upper glume as long as the spikelet, broadly ovate, convex, 5-nerved, margins hyalime, upper lemma bisexual, chartaceous, apiculate, palea similar its lemma, 2-keeled, stamens 3, grain reddish-brown, plano convex, granulate.

43. Paspalum scrobiculatum L., FBI 7: 10.1896; Bot. Bihar \& Orissa 1000. 1924; Gam vol. 3. 1772; Suppl. Bot. Bihar \& Orissa 176.1950; Bor 340.1960; GS. 422; HS. 524.

Perennial tufted, culms erect or decumbent, nodes glabrous, leaf blades linear-lanceolate, acuminate, margins white and scabrid, ligules membranous, short, sheaths compressed, keeled, racemes 2-3, spikelets 2 nate, single obovate or suborbicular, rhachis ribbon like, ciliate, pedicels flattened, ciliate, lower glume wanting, lower lemma nerved, epaleate membranous, upper glume membranous, glabrous concave, orbicular or broadly ovate, 7 nerved, upper lemma broadly ovate, turning brown with age, palea similar to its lemma, margins inflexed with flaps on either side at base, lodicules 2, quadrangular, stamens 3.

\section{Paspalum vaginatum Sw., Prodr. Veg. Ind. Occ. 21.1788; Bor 341. (Fig. 2C)}

Aquatic, perennial, rhizomatous, tufted; leaves linear-lanceolate, glabrous, margin puberulous, tip acuminate, sheath hairy, ligule membranous, young leaves 7-nerved, old leaves 1-nerved; spikes 2, sub digitate; spikelets 2-ranked, pedicelled, single, 2-flowered, lower floret empty and upper floret bisexual; pedicel glabrous; Spikelets planoconvex, glabrous; lower glume absent, lower lemma membranous, 1-nerved, it's palea 7-nerved, thick, stamens 2, orange, lodicules 2.

\section{Pennisetum americanum (L.) Leeke., FBI 7: 82.1986; Gam vol. 3.1792; Bor 350.1960; AP. Fl. 1236.}

An annual, cultivated, tufted grass, culms up to $2 \mathrm{~m}$ tall, nodes bearded, leaf sheaths overlapping, sparsely tubercled based hairs, ligule a ciliate rim, blades 30-90 × 0.5-5.0 cm, linear-lanceolate, base rounded, apex acuminate, pilose below, midrib prominent, panicle up to $25 \mathrm{~cm}$ long, spiciform, cylindric, dense; rhachis stout, villous below the inflorescence; involucral bristles $4 \mathrm{~mm}$, often purplish-coloured, spikelet $4 \mathrm{~mm}$, oblong, lower glume absent, upper glume minute, truncate, 3-nerved, lower lemma oblong, obtuse, 5-nerved, epaleate, upper lemma ovate-oblong, membranous, 2-nerved; palea very broad, truncate, ciliate at the tip, stamens 3, apex penicillate, stigmas plumose, caryopsis oblong-obovoid or fusiform; glabrous, top exposed.

46. Pennisetum pedicellatum Trin., Mém. Acad. Imp. Sci. Saint-Pétersbourg, Sér. 6, Sci. Math., Seconde Pt. Sci. Nat. 3(2): 184.1834; FBI 1: 86.1896; Gam vol. 3.1792; Bor 346.1960; AP. Fl. 1237.

An annual, tufted grass, culms up to $90 \mathrm{~cm}$ tall, branches from the base, leafy, leaf sheaths glabrous; ligule a shortly ciliate membrane; blades 10-20 × 3-9 cm, linear, flat, glabrous-sparsely hairy, racemes $13 \mathrm{~cm}$ long, cylindric, densely flowered, involucral bristles outer few, slender, short, $3 \mathrm{~mm}$, inner long, $1 \mathrm{~cm}$, numerous, densely villous below the middle, unequal, free from the base, spikelets $4 \mathrm{~mm}$, solitary in the involucral, sub sessile, lower glume very small, woolly; upper glume oblong-lanceolate, hyaline, apiculate; lower lemma oblong, truncate, 3-lobed, hyaline; upper lemma ovate-oblong, obtuse, apex fimbriate ciliate, coriaceous, shining; palea lanceolate, toothed.

47. Pennisetum polystachyon (L.) Schult., Gam vol. 3: 1792 (1241). 1934; Bor 346.1960.

Panicum polystachyon L., Syst. Nat. ed. 10. 870.1759; AP. Fl. 1238.

An annual, tufted grass, culms up to $40 \mathrm{~cm}$ tall, much branched, leaf blades $7.0-35 \times 0.5-1.5 \mathrm{~cm}$, panicle 20 $\mathrm{cm}$ long, linear, slender; rachis slender, puberulous; bristles densely plumose, especially the inner, spikelets solitary, rarely 2 in an involucre, pedicelled, lower glume minute or suppressed; upper glume puberulous; lower lemma similar to upper glume, 3-toothed; upper lemma chartaceous, fimbriate; palea similar to its lemma.

48. Pennisetum purpureum Schumach., Bor 348.1960; AP. Fl. 1238.

A perennial, stoloniferous grass, culms up to $2 \mathrm{~m}$ tall, erect, branching from lower nodes; nodes bearded, leaf sheaths 10-15 cm long, glabrous, ligule a dense fringe of hairs; blades 30-60 × 0.7-1.5 cm, linear-lanceolate, base rounded, margins scaberulous, apex acuminate, scabrid, midrib prominent, spikelike raceme up to $18 \mathrm{~cm}$ long, solitary, cylindric, yellowish or purplish; involucral bristles numerous of unequal length, one usually very much longer, $8 \mathrm{~mm}$, scabrid, ciliate, inner most scabrid, $6 \mathrm{~mm}$, sparingly plumose towards the base, spikelets solitary, sessile, if in fascicles $2-4$, lateral pedicelled, all lanceolate, $5 \mathrm{~mm}$, lower lemma absent or if present ovate-lanceolate, truncate, scabrid; upper glume triangular, margin incurved, 3-nerved, scabrid; lower lemma 
oblong-lanceolate, scabrid on outside surface, 5-nerved, male or barren; upper lemma lanceolate, rolled, scabrid at apex, 2-nerved, stamens 3, penicillate at apex, style united throughout, stigmas plumose, caryopsis ovoid.

49. Pennisetum setosum(Sw) Rich. FBI 7: 87.1896; Bor 340.1960.

Cenchrus strosum Sw., Prodr. Veg. Ind. Occ. 26.1788; AP. Fl.1239.

A perennial, tufted grass, culms up to $80 \mathrm{~cm}$ high, often fastigiately branched at the nodes, leaf sheaths glabrous; ligule with fringed long, soft hairs, blades 10-20 ×5-10 cm, linear, apex acuminate, slightly hairy; racemes $10 \mathrm{~cm}$ long, purplish brown, involucral bristles unequal, the outer not ciliate, short, $3 \mathrm{~mm}$, the inner longer, long silky hairy below the middle, $1.3 \mathrm{~cm}$, spikelet $4 \mathrm{~mm}$, solitary within the involucral, lower glume minute or completely absent; upper glume ovate-oblong, cuspidate, hyaline; lower lemma oblong, obtuse, bidentate, palea narrowly oblong, hyaline, male: upper lemma ovate-oblong, truncate, coriaceous, shining; palea oblong, truncate, toothed or ciliate at the apex, stamens 3.

50. Perotis indica (L.) Kuntze., Gam vol. 3.1814; Bor 611. (Fig. 2D)

Anthoxanthum indicum L., Sp. Pl. 28.

Perotis latifolia Ait., Hort. Kew Bull. 1: 96; AP. Fl. 1239.

Perennial, tufted, creeping or geniculately ascending from base; ligule absent, blades ovate-lanceolate, base amplexical, margin spinulose, tip acute; inflorescence spike like raceme, terminal, spikelets small, subsessile; glumes subequal, linear, 1-nerved, margin scabrid, awned, lemma and palea contains a bisexual floret, stamens 3.

51. Rottboellia exaltata (L.) L.f., FBI 7: 156.1896; Bot. Bihar \& Orissa 1059.1924; Gam vol. 3.1759; Suppl. Bot. Bihar \& Orissa 194.1950; Bor. 206; V. Fl. 211; MV. 207; HS. 525. (Fig. 2E)

Annuals, erect, tufted, clinging roots from lower nodes, nodes and inter nodes glabrous purple colour, leaf blades up to $60 \mathrm{~cm}$, much longer than inter nodes, acuminate tip, margins \& upper surface scaberlous, lower surface smooth, mid nerve prominent white, ligule small membranous, sheaths scaberlous; racemes cylindric, tapering towards the apex into an appendage, greenish yellow, spikelets 2 nate, sessile and pedicelled, sessile spikelet sunk in the receptacle, awnless, lower glume coriaceous, 2 keeled, up to 11-nerved, lower lemma male, ovate-lanceolate, 3-nerved, palea similar to its lemma, lodicules 2, stamens 3, upper glume boat shaped, chartaceous, upper lemma bisexual, hyaline, 1-nerved, palea hyaline, lanceolate, pedicelled spikelet green, 2 flowered, both are male, compressed.

52.Setaria parviflora (Poir.) M. Kerguelen, Lejeunia 120: 161.1987.

S. geniculata P. Beauv., Bor 360.

Perennial herb, tufted, rhizome short; culms short, flat, procumbent, up to $25 \mathrm{~cm}$ high; leaf sheath purple, blade $10 \mathrm{~cm}$ long, $1 \mathrm{~cm}$ wide, margin purple, ligule membranous; inflorescence panicle cylindric, dense, 4.0$4.3 \mathrm{~cm}$ long; spikelets single, if 2-nate, lower spikelets reduced to bristles (about 10), upper half part purple; spikelets ovate, planoconvex, middle part of the pedicel hirsute, lower glume small 3- nerved, half of the spikelet, lower lemma membranous margins inflexed, epaleate, empty; upper glume membranous, upper lemma boat shaped, crustaceous rugose, it's palea similar, containing a bisexual floret, stamens 3 .

\section{Setaria pumila (Poir.) Roem \& Schult., Gam vol. 3.1789; Bor 363; AP. Fl. 1254. (Fig. 2F)}

Annual tufted grass, culms up to $50 \mathrm{~cm}$ high, nodes glabrous; leaf sheaths glabrous, ligule a fringe of hairs, blades linear-lanceolate, flat, base cordate, apex acuminate; panicle spiciform, cylindric, densely flowered, spikelets ovoid; lower glume, upper glume and higher lemma ovate, hyaline; upper lemma crustaceous, transversely rugose, stamens 3 .

54. Setaria verticellata (L.) P. Beauv., FBI 7: 80; Gam vol. 3.1789; Bor 365.

Panicum verticillatum L., Sp. Pl. ed. 2.82; AP. Fl. 1255.

Annual, tufted, 2-4 culms in a tuft, ererct, culms up to $2 \mathrm{~m}$ high; stem flat, nodes swollen, straite, solid; leaves up to $40 \mathrm{~cm}$ long, $3 \mathrm{~cm}$ wide, linear, base narrow, upper surface scabrid, 10 side nerves, lower surface white, no nerves, margin scaberulous, ligule small membranous; inflorescence 20-23 cm long, rhachis ends with a setae, lower 3 spikelets 3-nate, remaining all 2-nate, spikelet having 1-long setae, setae downward barbelleate. 

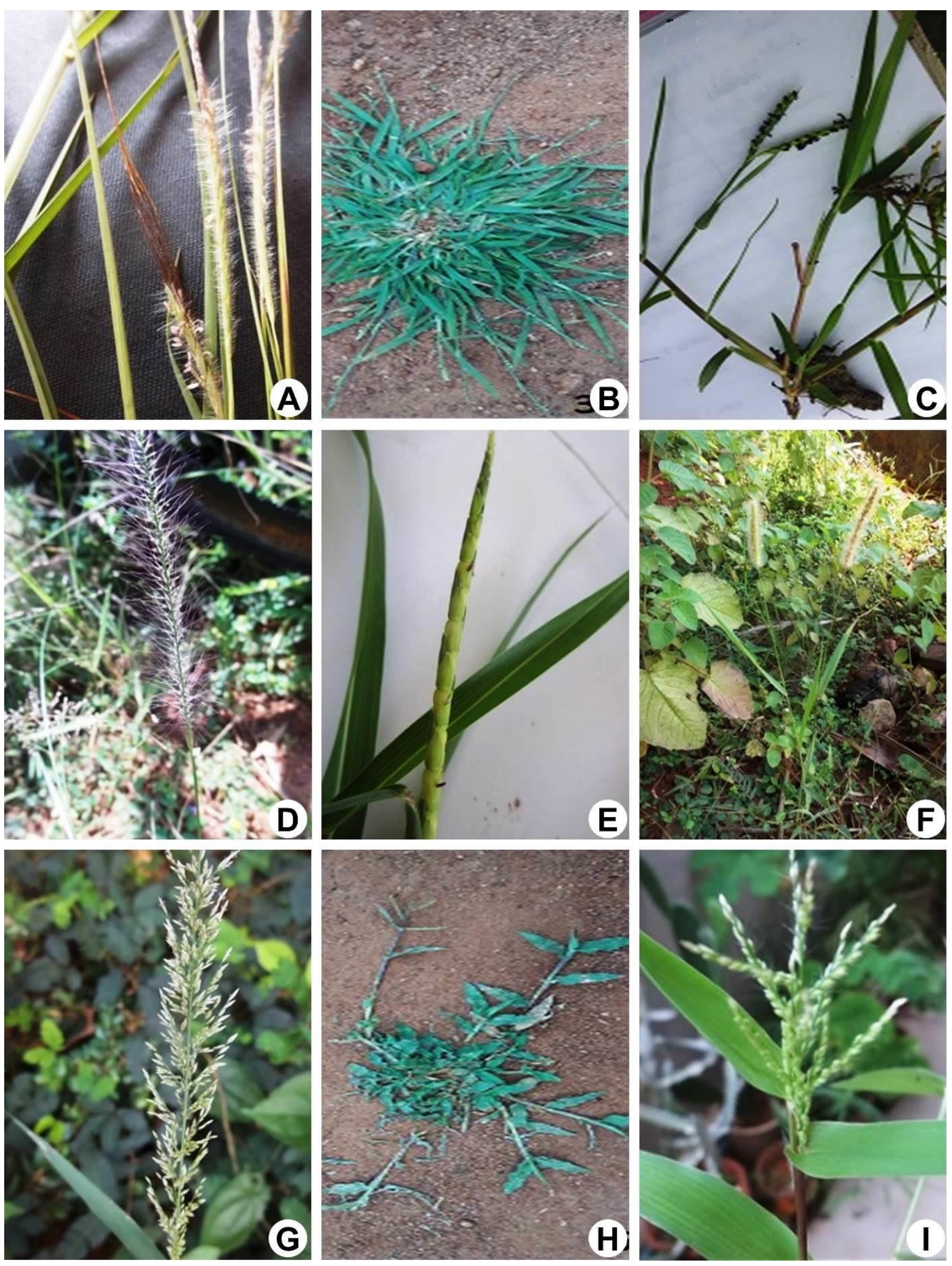

Figure 2. A, Heteropogon contortus (L.) Beauv. ex Roem. \& Schult.; B, Paspalidium flavidum (Retz.) A. Camus.; C, Paspalum vaginatum Sw.; D, Perotis indica (L.) Kuntze.; E, Rottboellia exaltata (L.) L.f.; F, Setaria pumila (Poir.) Roem \& Schult.; G, Sporobolus indicus (L.) R.Br.; H, Urochloa panicoides P.Beauv.; I, Urochloa setigera (Retz.) Stapf.

55. Sporobolus coromandelianus (Retz.) Kunth., FBI 7: 252.1896; Gam vol. 3: 1817 (1258). 1934; Bor 627.1960 .

Agrostis coromandeliana Retz., Obs. Bot. 4: 1786.

Sporobolus commutatus (Trin.) Kunth, Enun. Pl. 1: 214.1833.

www.tropicalplantresearch.com 
Vilfa commutate Trin., Diss. Bot. 156.1824; AP. Fl. 1258.

Annual, tufted grass, decumbent, nodes glabrous, ligule membranous, blades lanceolate, flat, base cordate, margins cartilaginous spinulosely tooth, apex acute, sparsely tuberculate based hairs, inflorescence panicle, decompound, branches capillary, horizontal, whorled at base, spikelets minute, pedicelled, lower glume minute, suborbicular, upper glume, lemma similar, palea hyaline contains bisexual floret, stamens 3, caryopsis ellipsoid.

56. Sporobolus indicus (L.) R.Br., FBI 7: 247.1896; Gam vol. 3.1817; Bor 629.1960; AP. Fl. 1259. (Fig. 2G)

Perennial, tufted grass, culms $60 \mathrm{~cm}$ high, nodes, nodes glabrous, leaf sheaths glabrous, ligule fringe of hairs, leaf blades linear flat, base truncate, apex acuminate, inflorescence contracted panicle, branches filiform, spreading, spikelets small, single flowers, lower glume minute, ovate, hyaline, upper glume broadly elliptic, hyaline, acuminate, lemma lanceolate, its palea small hyaline, stamens 2, stigmas plumose, caryopsis obovoid.

57. Themeda laxa (Anderson) A. Camus., Gam vol. 3.1746; Bor 251. AP. Fl. 1264.

Perennial, culms up to $40 \mathrm{~cm}$ high, tufted; leaf blades filiform, apex acuminate; panicle up to $8 \mathrm{~cm}$ long, leafy, few racemes, shortly peduncled outer spathes $2.5 \mathrm{~cm}$, proper spathes glabrous, longer than the spikes; involucral spikelet: 4, all on the same level, male; lower glume glabrous except few bristles near the apex; pedicelled spikelets: similar to involucral spikelet; sessile spikelet: solitary, bisexual, awned.

58. Themeda quadrivalvis (L.) Kuntze., Bot Bihar \& Orissa 1050.1924; Gam. vol. 3: 1746.1934; Bor 252; FBI 7: 213.1896; GS. 442; HS. 528; MV. 2008; V. Fl. 210.

Annual, erect, culms and nodes glabrous, leaf blades flat, linear lanceolate, tip acuminate, rounded at base, mouth ciliate, ligule membranous; inflorescence leafy panicle, fascicled, erect, spatheoles boat shaped acuminate, tip 3-5 nerved, involucral spikelets pairs situated at the same leaf, middle sessile spikelet awned, contains bisexual floret remaining four spikelets sessile, contains male florets, stamens 3, glumes of male florets 2 keeled with scarious and ciliate margin on one side, pedicelled spikelets 2 , linear lanceolate, contains male floret or empty.

59. Themeda triandra Forssk., Gam. vol. 3: 1746.1934; Bor 254: FBI 7: 211.1896.

Themeda imberbis (Retz.) Bomb., Fl. 2: 993; Bot. Bihar \& Orissa 1049.1924.

Perennial, erect, up to $170 \mathrm{~cm}$ high, culms \& nodes glabrous, leaf-blades flat, up to $41 \mathrm{~cm}$ long, linearlanceolate, acute, ligule membranous, rounded, truncate ciliate; inflorescence up to $40 \mathrm{~cm}$ long, panicles leafy fascicled, erect or drooping, spathes long, leaf like, acuminate, margins with long hairs, involucral spikelets 2 pairs situated at the same level, shortly acuminate, each pair contains 5 spikelets, middle one sessile awned and contains bisexual floret, callus bearded, sessile spikelets, surrounded by 4 male spikelets, involucral spikelets; lower glume reddish brown, membranous, 2-toothed at apex, teeth unequal, 2-keeled with scarious margins on one side, 11-nerved, covered with stiff bristles, upper glume margins ciliate in the upper half, 3-nerved, 2keeled, lemma male, hyaline, obtuse, margins ciliate, 1-nerved, epaleate, stamens 3, sessile spikelets, lower glume coriaceous truncate at apex, 9-nerced, upper glume coriaceous, obtuse, 3-nerved, lower lemma hyaline, epaleate, upper lemma reduced to an awn, epaleate, pedicelled spikelets male, pedicels glabrous.

60. Urochloa panicoides P. Beauv., Gam vol. 3: 1775; Bor 372. FBI 7: 35 p.p; HS. 530; V. Fl. 214. (Fig. 2H)

Annual, tufted, herbs sub erect, nodes swollen; leaves hairy, ligule fringe of hairs or glabrous, cordate at the base, leaf sheath clasping the stem; inflorescence panicle, racemes 5-6, spikelets 2-nate, spikelets ovate to lanceolate, glumes membranous, unequal, upper lemma cuspidate.

61. Urochloa setigera (Retz.) Stapf., Fl. Trop. Africa 9: 598.1920; Bot. Bihar \& Orissa 1003.1924; Gam. vol. 3: 1775. (Fig. 2I)

Panicum setigerum Retz., Obs. Bot. 4: 15.1786; FBI 7: 36.

Brachiaria setigera (Retz.) Hubbard in Hooker's Icon. Pl. 34. sub. t. 3363.1938; Suppl. Bot. Bihar \& Orissa 177.1950; Bor 286.

Annuals up to $75 \mathrm{~cm}$ high, culms decumbent, rooting at lower nodes, geniculately ascending, nodes pubescent, leaf sheaths hairy, leaf blade lanceolate, $12-14 \mathrm{~cm}$ long, wide $2.6 \mathrm{~cm}$, leaf base cordate, acute tip, ligule a ring of white hairs; inflorescence panicle, 10-15 cm long, racemes distant 10-20, crowded 2-3, 2-6 cm long, rhachis triquentrous, hairy on the angles interposed with 3-5 long hairs, each spikelet bears 3-5 long hairs, 
spikelets 2 nate, sessile and pedicelled, spikelets $0.3 \mathrm{~cm}$ pedicels pubescent, glumes dissimilar, lower glume one fourth of the spikelet, pink.

\section{CONCLUSION}

Uncultivated grasses are drought tolerant, fast growing, disease resistant, no need to sow, no need to watering, no need to use fertilizers because these soils enriched with humus (cattle dung), nutrients and moisture. They are helpful in controlling soil erosion. Over grazing leads the pastures to disappear, so retire the land from the use by livestock at least till the green cover is sufficiently restored.

\section{REFERENCES}

Achariyar KR \& Mudaliyar CT (1921) A Handbook of South Indian Grasses. Madras.

Blatter E \& McCann C (1935) The Bombay Grasses. Sci. Monogr. No. 5. Imp. Counc. Agric. Rec. India.

Bor NL (1960) The Grasses of Burma, Ceylon, India and Pakistan. Pergamon Press, London.

Cooke T (1901-1908) The Flora of the Presidency of Bombay. London.

Fischer CEC (1934) Gramineae in J.S. Gamble's Flora of Madras. London.

Gamble JS (1896) The Bambuseae of British India. Annals of Royal Botanical Garden Calcutta 7(1): 1-133.

Hooker JD \& Stapf O (1896) Flora of British India, Vol. 7. Gramineae International, U.K.

Sriramulu HS (1986) Flora of Srikakulam district, Andhra Pradesh, India (Flora of India series). Indian Botanical Society, Meerut.

Subba rao GV (1977) Flora of Visakhapatnam district, Andhra Pradesh, India (Flora of India series). Indian Botanical Society, Meerut.

Venkaiah M (2004) Studies on the Vegetation and Flora of Vizianagaram District, Andhra Pradesh. Andhra University, Visakhapatnam. 